\title{
EFFORTS TO PROGRESS IN THE HARMONIZATION OF L2 PSA DEVELOPMENT AND THEIR APPLICATIONS IN EUROPE. STATUS OF ACTIVITIES AND PERSPECTIVES AFTER THE FUKUSHIMA ACCIDENT
}

\author{
E. RAIMOND \\ Nuclear Safety Division, Severe Accident Department \\ BP 17, 92262 Fontenay-aux-Roses, France \\ E-mail : emmanuel.raimond@irsn.fr \\ Received May 12, 2012
}

A major issue for all nuclear stakeholders is to keep the probability of circumstances that could lead to core damage as low as possible. In addition, for NPP, appropriate accident management provisions are to be implemented to limit the consequences associated with an accident.. Development and application of L2 PSA is a structured way to demonstrate that such objectives are achieved. The paper presents the efforts recently done in Europe to harmonize some best-practices in that field, from research area to risk assessment. The Fukushima Daiichi accident reiterated the importance of these activities and the need to efficiently reinforce the NPP safety based on risk assessment conclusions. New perspectives in Europe are briefly presented.

KEYWORDS : NPP, Severe Accident, Risk Assessment, L2 PSA, European Framework Programme, ASAMPSA2, SARNET

\section{INTRODUCTION}

Most of the existing Nuclear Power Plants (NPPs) are designed with the principles of defence-in-depth and incorporate strong containment and engineering systems to protect the public against radioactive release for a series of postulated accidents.

Nevertheless, in some very low probability circumstances, severe accident sequences may result in core melting and plant damage leading to dispersal of radioactive material into the environment and thus constituting a health hazard to the public.

A major issue for all stakeholders is to keep the probability of such circumstances as low as possible. In addition, appropriate accident management measures, allowing an efficient limitation of the consequences of such events, should now be implemented, as recommended for example by WENRA reference levels.

Following the initial US effort in the 80's, in most countries, level 1 and level 2 Probabilistic Safety Assessments (L1 and L2 PSA) have now been developed for the existing and future plants and are used to demonstrate that the probability of occurrence of a severe accident is low enough and that, if such an accident were to occur, all reasonable provisions would be taken to limit the conse- quences. These studies, updated in function of plant modifications, new knowledge and scope extension, contribute to the continuous improvement of plants safety, while identifying remaining dominant risks.

Nevertheless, regarding the severe accident phenomenology, the remaining uncertainties, and also the diversity of accident scenarios considered, the development of L2 PSA is still a very complex activity often conducted by rather small teams. In parallel, the expectation of these studies is very large, for example:

- validation of severe accident measures (SAM),

- chieving safety goals or acceptability of the level of risk,

- cost-benefit analysis,

- support for decision regarding plant life extension,

- identification of R\&D needs for closing issues,

- capitalization of knowledge,

- emergency preparedness ...

Such expectations require robust and validated studies. But one should recognize that, in some cases, discrepancies may exist between the real quality of the L2 PSAs (regarding the complexity of the different issues) and the expected applications. For this reason, the L2 PSAs are generally used very carefully in their applications. 
The need to share experience in the development and the application of L2 PSA in the international accident management community has been continuously expressed. The development of standards, best-practice guidelines, and state-of-the art methods are useful guidelines for allowing experts to share their experiences and to formalize agreed upon best-practices.

This paper provides information on recent European initiatives regarding the harmonization of the assessment of severe accident risks and its applications: the European Advanced Safety Assessment Methodologies: Level 2 PSA (ASAMPSA2) project.

The importance of such activities on guidance is then commented in relation with/to the Fukushima NPP accidents which has highlighted, once again, the need for high quality and complete PSAs and a credible decision making process to implement, if needed, additional NPP safety enhancements able to limit accident consequences.

\section{TWO MAJOR PROJECTS WITHIN THE EUROPEAN FRAMEWORK PROGRAMMES}

\subsection{SARNET (Severe Accident Research NETwork of Excellencel}

In the European context, the Severe Accident Research NETwork of Excellence (SARNET [1,2]) gathers a large part of activities concerning severe accident issues. SARNET was initiated in 2004 with 51 organisations involved in severe accident research in Europe plus Switzerland and Canada. A second project, named SARNET2, was launched in 2009 that gathered, 47 organizations from 24 countries (Europe plus Switzerland, Canada, USA, Korea, India and Japan).

SARNET2 includes some integration activities (database on experimental results, integration of knowledge in the ASTEC IRSN-GRS integral code, extension of ASTEC to BWR and CANDU reactors), spreading of knowledge (conferences, seminars, courses, encouragement of exchange of students ...) and some research activities considered with a high level of priority (in-vessel core coolability, ex-vessel melt-pool configuration during molten core concrete interaction (MCCI) and coolability by top flooding, hydrogen mixing and deflagration/ detonation in containment, melt relocation into water and fuel coolant interaction (FCI), ruthenium behaviour and iodine chemistry in reactor cooling system and containment).

Some activities concerning L2 PSA were performed within the first project in 2004-2008 (general methodology, uncertainties assessment and dynamic reliability methods [3]) and have been used to define and initiate the ASAMPSA 2 project of the $7^{\text {th }}$ Framework programme that is described hereafter. Technical exchanges between SARNET and ASAMPSA2 have continued in particular on the update of the knowledge of the severe accident physical phenomena and management measures and on the L2 PSA requirements for computer codes such as ASTEC.

\subsection{ASAMPSA2 (Advanced Safety Assessment Methodology: Level 2 PSAl}

Within the European community responsible for fission reactor safety (plant operators, plant designers, Technical Safety Organizations (TSO), Safety Authorities), a need to develop best practice guidelines for the level 2 PSA methodology has been repeatedly expressed, with the aim of both fulfilling the requirements of safety authorities in an efficient way, and also promoting harmonization of practices in European countries in order to use results from level 2 PSAs with a greater confidence.

Many existing guidelines, like those developed by the IAEA, propose a general stepwise procedural methodology, mainly based on the US NRC's NUREG-1150 study and high level requirements (for example on assessment of uncertainties). While it is clear that such a framework is necessary, comparisons of existing level 2 PSA, performed and discussed in the SARNET L2 PSA group, have shown that the detailed criteria and methodologies of current level 2 PSAs strongly differ from each other in some respects. Currently in Europe, integration of probabilistic findings or insights into the overall safety assessment of NPPs is quite differently understood and implemented.

Within this general context, the project objectives were to highlight common best practices, develop the appropriate scope and criteria for different level 2 PSA applications, and promote optimal use of the available resources. Such a common assessment framework was expected to support some harmonized view on nuclear safety, and to help formalize the role of probabilistic safety assessment.

Noteworthy considerations include:

- PSA tool should be fit for purpose in terms of quality of models and input data,

- the scope should be appropriate to the life stage (e.g. preliminary safety report, pre-operational safety report, living PSA) and plant states (e.g. full power, shutdown, maintenance) considered,

- the objectives, assessment criteria and presentation of results should facilitate the regulatory decision making process.

One important characteristic of this coordinated action was to bring together different stakeholders (plant operators, plant designers, TSO, Safety Authorities, PSA developers), regardless of their role in the safety demonstration and analysis.

The scope of the project only covers the internal initiating events with a particular effort on meaningful assessment of uncertainties.

The project started at the beginning of 2008 with a 3 years duration. 22 organizations from 13 European countries 
have participated to the project; coordinated by IRSN. It was then extended by one year until the end of 2011. A final open workshop has been organized in March 2011, $7^{\text {th }}$ to $9^{\text {th }}$, to discuss the review of the guideline. The final publication of the guidelines will be released mid 2012 because most of the project partners were involved in the answer of their country to the $11^{\text {th }}$ of March Fukushima event. The guidelines will be a public document and will be available on the project website [4].

\section{SOME OUTCOMES OF THE ASAMPSA2 PROJECT}

\subsection{General}

\section{a) Objectives}

The aim of the coordination action was to build a consensus on the L2 PSA scope and on methods deemed to be acceptable, according to the different potential applications. It was clear from the beginning that, depending of the issues, there is a range of outcomes that can be considered acceptable.

To represent this range, the project initially tried to distinguish between a 'limited-scope' methodology and a 'full-scope' one, based on what is currently technically achievable in the performance of a level 2 PSA.

The notion of 'limited-scope' methodology may correspond to the case where the study is performed to answer some precise question (for example the quantification of $\mathrm{LERF}^{1}$ ), allowing simplification of some parts of the study, and limitation of the needed resources and delay.

The distinction between limited-scope and full-scope methodologies has been widely discussed in the initial phase of the project and the possibility to establish two separated guidelines has been examined. But from a practical point of view, it appeared that many variations in the definition of what is a 'limited-scope study' exist in relation with the different applications. Consequently, the partners of the project have decided to build a unique document including all issues related to level 2 PSA development and applications. For each issue, the different level of details and acceptable methods are described with some recommendations.

It was also stated that the guidance on applications of L2 PSA was a crucial part but also the most difficult to establish. It has conducted the project partners to build a specific volume of the guidelines on this topic.

b) Relationship with the L2 PSA "End-Users"

In designing the ASAMPSA2 project, the relationships with the L2 PSA 'End-Users' (establishing the needs of

${ }^{1}$ LERF : Large Early Release Frequency the 'End-Users' for the performance of a L2 PSA as well as assuring the acceptance of the guidelines to be prepared at the end of the project by a majority of the 'End-Users') were considered as a key point. A dedicated working group, coordinated by PSI, has been established to help in formalizing these relationships.

These elements were planned in two steps in the project. At the beginning of the project, a survey was conducted to establish more precisely the needs of the 'End-Users' community regarding many aspects of performing a L2 PSA. The results of the survey were discussed during a dedicated workshop, hosted by Vattenfall in Hamburg (Germany) in October 2008. At the end of the project, an external review of the guidelines has been organized to receive the response from the End-Users community. The review has been discussed during a workshop hosted in March 2011 by Fortum in Helsinki (Finland). This final review, like the initial survey, has associated European stakeholders and also organizations from other countries, especially those members of the OECD CSNI-WG-Risk.

ASAMPSA2 has taken into consideration the positions provided by End-Users irrespective of their role (plant operators, plant designers, Technical Safety Organizations (TSO), Safety Authorities).

c) Link with the international scientific research activities related to severe accidents

A level 2 PSA is mainly based on a set of deterministic studies on the different phenomena related to severe accident progression. A large part of the guidelines concerns the way of quantifying each part of the accident progression.

The first draft of the different chapters has gathered the methodologies currently used by the partners PSA experts and describe some rationale. To improve its final quality regarding the state-of-art for each topic, the guidelines have been open for review by specialists involved in the SARNET Network of Excellence or NEA/CSNI/ WGAMA members.

d) Link with other existing standards

Others countries, outside the European Union, or international organizations have developed some L2 PSA guidance. The ASAMPSA 2 guidelines provide a number of key references from OCDE, IAEA or countries that can be useful. In many cases, examples of good practices have been extracted from these references.

\subsection{Some Outcomes of the Initial End-Users Survey}

Feedback on the 2008 End-Users survey helped in the identification of some technical issues where harmonization or best-practices are particularly needed, e.g.:

- L1 PSA - L2 PSA Interface: advantages and disadvantages of the integrated and non integrated studies, use of L1 PSA probabilistic tools or dedicated tools for L2 PSA,

- methods for uncertainty assessment (issue by issue, in the event tree, propagation, for results presentation), 
may depend on the L2 PSA objectives, plant design and may be limited to some relevant issues (the assessment of all uncertainties is not reasonable ...),

the closure of issues in accident progression regarding research activities: in that context, an issue is 'closed' when L2 PSA developers find enough knowledge or validated codes for the assessment of risks (it can be dependent on the plant design),

- the assessment of initial containment leakage, use of historic data (tests), assessment of containment isolation failure ...

The End-Users survey also shows that there is a lack of uniformity between the countries in the objectives and applications of L2 PSAs. Only a few EU Safety Authorities have precise safety goals regarding severe accidents, and in general the legislation or rules, when they exist, are not strictly applied. Very few utilities have a voluntary approach for 'risk-informed' application of L2 PSA (Finish utilities as mandated in legislation, EDF recently developed application for periodic safety review). Some utilities still have an unclear view on how and why to develop a L2 PSA.

It is expected that the ASAMPSA2 project will help in harmonization of technical issues by providing a global (but practical) vision of how the different risks can be assessed within a L2 PSA taking into account the existing knowledge and codes. It should also help in harmonization on application of L2 PSA, in particular to help to identify some plant 'risk reduction options'.

\subsection{Structure of the Guideline}

At the end of the project, the ASAMPSA2 guidelines are composed of three volumes.

The first volume includes a general description of L2 PSA content and structure and discusses the different possibilities to present the L2 PSA results depending on applications. The project has used (as much as possible) information available on public domain, mainly from other international collaboration initiative, for example on the description of safety criteria.

The second volume of the document contains all technical recommendations gained from the experience of the ASAMPSA2 partners and external sources, for Gen II and III NPPs. It covers the methodological topics (level 1level 2 PSA interface, Human Reliability Assessment, the event tree structure, the uncertainties assessment ...), the quantification of severe accident progression and containment loading, the containment performance (tightness), the plant system behaviour in severe accident conditions and the source term assessment. This volume shows the very large number of issues that must be examined in a L2 PSA and discusses topics by topics uncertainties assessment. The different topics were distributed among the partners, depending on their experience, and based on resources available for this project.

The last volume of the guidelines concerns the appli- cations for Gen IV reactors, with the objective to describe how far the existing recommendations for Gen II and III reactors L2 PSA may apply for the Gen IV reactors concepts. It raises some key issues for Very-High Temperature Reactors (VHTR), Sodium-cooled Fast Reactors (SFR), the Gas-cooled Fast Reactors (GFR) and Leadcooled Fast Reactors (LFR). This volume can be used a starting point for L2 PSA development for these reactors. The development of this volume was coordinated by CEA.

\subsection{Outcome of the Final End-Users Survey and Further Needs}

The End Users Survey has provided number of comments and proposal for improvements of the draft version of the ASAMPSA2 guideline.

\section{- Relevance of the ASAMPSA2 guidelines regarding recent $R \& D$ results}

It is apparent that respondents appreciated the precision and level of details of the ASAMPSA2 guidelines, which can be considered as a precious handbook for the L2 PSA developers. The scientific community, and in particular SARNET representatives, have indicated that for severe accident phenomenology, no major point was missing or wrongly addressed in the guideline. They have also provided some complements for some issues. The guidelines will be also used as one of the technical bases for the preparation of next plan of activities of SARNET.

\section{- Expectations from L2 PSA developers on additional $R \& D$ activities}

The ASAMPSA2 guidelines show that knowledge and simulation tools are in place for most severe accident phenomena but expectations for R\&D progress have also been identified, for example: method for introduction of recovery actions in L2 PSA, core degradation for shutdown states with open reactor vessel (air ingress), in-vessel and ex-vessel degraded core coolability, positive and negative impact of in-vessel water make-up, kinetics and amplitude of release in case of basemat penetration, reduction of uncertainties of source term prediction, steam explosion (corium explosibility, real status of corium in the vessel which may be too cold for explosion, capabilities of codes to accurately predict the behaviour of structures ...), accurate reactivity accident modelling ...

\section{- General comments}

The major points of the discussions during the external review concerned the objectives of the document.

Firstly, the ASAMPSA2 guidelines were at times deemed broad by certain respondents, because the guidelines rarely proposed a single solution, but instead often proposed several possibilities to build a L2 PSA. This status is in fact consistent with the direction taken by the ASAMPSA2 partners: to identify a panel of acceptable methods but not to impose a unique set of procedures.

Nevertheless, the perspective to derive a shorter document from the existing ASAMPSA2 guidelines that 
could be endorsed by both regulators group (ENSREG or WENRA) and utilities group (ENISS) has been identified as a useful follow-up action.

Secondly, some reviewers have regretted that the ASAMPSA2 guidelines do not cover the accident initiated by external events. This was not in the objectives of the project as defined with the partners and the European Commission and was identified as a possible follow-up activity in Europe. The Fukushima accident has of course reinforced this conclusion.

\section{- Further needs}

A set of possible follow up activities has been identified. It includes, for example:

- improvement of tools able to incorporate deterministic simplified model to simulate accident progression in an event tree, to calculated source term assessment ; such tools should include Monte-Carlo simulations and offer possibilities for dynamic reliability methods,

- improvement of tools and methodologies to interface L1 and L2 PSA,

- extension of the ASAMPSA2 guidelines to external events, shutdown states,

- testing the guidelines on one application,

- elaboration of L2 PSA standardized risk metrics that could be applied for all plants,

- elaboration of database on experimental results useful for L2 PSA developers,

- use L2 PSA for validation of severe accident management options,

- training and review services,

- ...

\section{LESSONS FROM THE FUKUSHIMA ACCIDENT FOR THE L2 PSA DEVELOPMENT AND APPLICATIONS}

Before discussing any lessons from the Fukushima accident, we have to recognize and emphasise the merits and courage of all Japanese teams and individuals who have worked in adverse conditions to minimize as far as possible the consequences of this huge tsunami.

The following comments concern the role of L2 PSA in the continuous process of plant safety improvement. It seems that the Fukushima accident reveals an example where the existing PSAs have not conducted to some safety reinforcements needed on the Fukushima Daiichi site:

- the frequency of the initiating event (earthquake and tsunami) seems to have been underestimated,

- the robustness of the Fukushima Daiichi units 1, 2, 3 and 4 in case of long term total loss of electrical supply and cooling, and the capabilities of their containments to limit the radioactive release were insufficient.

Moreover, this accident has shown the difficulty in managing multi-unit accidents and also the dependency between the management of reactors and spent fuel pools (SFP). These risks are in general not addressed in L2 PSA when they obviously should be.

It can also be noted that the management of the SFP of unit 4 has been extremely difficult due to the dependencies with the other units, even if the unit 4 was in shutdown state. It seems clear today that risks associated with spent fuel pool must be systematically addressed by PSA.

These facts prove once again how important is the development of complete risk assessment analysis for all initiating events (included all types of external events), all states of reactors, all sources of radioactive material (reactors, SFPs,...) and dependencies between units for accident management.

The lesson could be compared to the situation in Europe at the beginning of the ASAMPSA2 project when the End-User survey showed that a decision making process, based on L2 PSA conclusions, was not in place in many European countries. This is being evolving with development of L2 PSA in most countries and introduction of severe accidents topics in the Periodic Safety Review process.

\section{TOWARDS A NEW EUROPEAN PROJECT : ASAMPSA_E}

As mentioned above, after the ASAMPSA2 project, some follow-up projects that could be useful for European nuclear stakeholder were identified. After the Fukushima accident, the European Commission has submitted a call of tender to promote a project making the link between PSA and extreme external events:

The nuclear accident in Japan resulted from the combination of two correlated extreme external events (earthquake and tsunami). The consequences (flooding in particular) went beyond what was considered in the initial NPP design. Such situations can be identified using PSA methodology that complements the deterministic approach for beyond design accidents. If the performance of a Level 1-Level 2 PSA concludes that such a low probability event can lead to extreme consequences, the industry (system suppliers and utilities) or the Safety Authorities may take appropriate decisions to reinforce the defence in depth of the plant. The present topic aims at providing best practice guidelines for the identification of such situations with the help of Level 1-Level 2 PSA and for the definition of appropriate criteria for decision making in the European context. Involvement of regulatory authorities in the foreseen action is a must. Cooperation with Japan is welcome.

22 European organizations (from 17 countries) have submitted a 3 years project, called ASAMPSA_Extended, with a format close to ASAMPSA2, including the following activities: 
- Guidance for practicable and meaningful method to introduce external events in L1-L2 PSAs,

- Guidance for a decision making process based on extended PSAs,

- Additional guidance for L2 PSA (SAM validation, complements for shutdown states of reactors ...)

The outcome of this project should help European stakeholders to verify, by means of probabilistic approaches (with PSA extended to cover risk contribution from all sources of radioactivity, all operating states for all important sources of radioelements and all types of initiating events (internal events, internal hazards and external hazards)), the NPPs safety robustness, after having included the additional improvements defined in response to the Fukushima Daïchi accident and the stress test process conducted in all European countries.

The precise content of the project has to be discussed with EC. It should provide a useful framework for further improvements of L2 PSA quality and impact of NPP safety.

\section{CONCLUSIONS}

The present paper presents an overview of European activities on harmonization of probabilistic risk assessment regarding severe accidents at NPPs.

These harmonization activities appeared useful within a perspective of continuous plants safety improvement in all countries, especially for existing plants which are subject in many countries to plant life extension programs.

This harmonization can progress at different levels:

$\rightarrow$ on high level safety requirements that should find place in national regulation,

$\rightarrow$ on the fundamental analysis of the severe accident phenomena as provided within SARNET or OECD activities,

$\rightarrow$ through the comparison and sharing of experience in L2 PSA development and applications (e.g. OCDECSNI WG-Risk activities),

through the development of L2 PSA best-practice guidelines or standards as conducted recently within the EC ASAMPSA2 project ; it offers a structured framework to discuss in detail how to make the best use of existing knowledge and codes for the quantifi- cation of risks,

- through international reviews.

These activities at each level are ultimately useful and help stakeholders to make risk assessments more robust, and to identify or confirm plant risk reduction options and severe accident measures.

The Fukushima accident has emphasized the importance of these activities including the decision-making process.

The ASAMPSA2 project has also shown that fundamental knowledge and codes are available for most part of accident analysis and that further activities should concern mostly PSA scope extension and validation of designed accident management provisions.

Many plant safety reinforcements should be achieved in the next few years after the post-Fukushima stress test process in Europe. Full-scope PSAs should then be used to confirm the design choices.

\section{ACKNOWLEDGEMENTS}

The Author thanks all partners involved in the ASAMPSA 2 project from IRSN, GRS, NUBIKI, TRACTEBEL, FKA, CCA, FORTUM, ENEA, PSI, VGB, IBERDROLA, UJV, VTT, RSE, AREVA, AMEC, CEA, STUK, NRG, SCANDPOWER, all external reviewers, from Europe and outside (US, Korea, India, Japan, ...) and also Michel Hugon, scientific officer from the European Commission who has supported this ASAMPSA2 project.

\section{REFERENCES}

[ 1 ] J.-P. Van Dorsselaere, A. Auvinen, D. Beraha, P. Chatelard, C. Journeau, I. Kljenak, A. Miassoedov, S. Paci, W. Tromm, R. Zeyen, "The European Research on Severe Accidents in Generation-II and-III Nuclear Power Plants", Science and Technology of Nuclear Installations, Volume 2012, Article ID 686945, doi:10.1155/2012/686945

[2] J.-P. Van Dorsselaere, A. Auvinen, D. Beraha, P. Chatelard, C. Journeau, I. Kljenak, A. Miassoedov, S. Paci, W.Tromm, R. Zeyen,"Some outcomes of the SARNET network on severe accidents at mid-term of the FP7 project", Proceedings of ICAPP 2011, Nice, France, May 2-5, 2011, Paper 11369

[3] B. Chaumont, E. Raimond \& al, L2 PSA methods harmonization, ERMSAR conference, Nesseber, Bulgaria, September 23-24, 2008,

[4] www.asampsa2.eu 